\title{
The Emotional Conduction and Impact Discovery of the Mass Emergencies
}

\author{
Guo-Qiang Xiong ${ }^{1, a}$, Hao Wang ${ }^{2, b, *}$ \\ ${ }^{1,2}$ School of Economics and Management, Xi'an University of Technology, Xi'an, China \\ axgq168@163.com, bwhxalg091@126.com \\ ${ }^{*}$ Corresponding author
}

Keywords: Mass emergencies, Emotion, Conduction.

\begin{abstract}
As one of the important factors of Mass Emergencies, emotion plays an important role in the evolution of Mass Emergencies. In this paper, the model of emotional conduction has been built, by emotional experiments and mathematical statistics, to analysis the effect of emotion on Mass Emergencies behavior. Research shows that (1) The presence of highly related to emotion and participants risk perception. Emotion will enable participants risk cognition deviation from the actual, and impact the participants behavior decision.(2) The emotion has the characteristics of ebp, periodicity and Infectious. The characterization of factors are highly related to the head feeling.(3) Pessimism is easy to make the participants take violence to show their own appeal, optimism will enable participants tend to conservative decision behavior. The results of these studies have an important significance in the development and evolution of Mass Emergencies. Introduction (Heading 1). All manuscripts must be in English, also the table and figure texts, otherwise we cannot publish your paper. Please keep a second copy of your manuscript in your office. When receiving the paper, we assume that the corresponding authors grant us the copyright to use the paper for the book or journal in question. Should authors use tables or figures from other Publications, they must ask the corresponding publishers to grant them the right to publish this material in their paper.
\end{abstract}

\section{Introduction}

The dislocation of the distribution of benefits accelerated the change of structure of Chinese society and the pattern of interests. The intensified Contradiction between the various interest groups lead the mass incidents conflict frequently. Not only caused economic losses of property, but also led to social and psychological fear and insecurity. To some extent, the conflicts weaken the government's credibility, seriously affecting the Social stability. Therefore, mass incidents becoming a popular area, the academic use a variety of methods to explore the factors of mass emergencies, including population size [1], the social structure [2], action strategies [3], information dissemination [4] and evolution mechanisms [5]. Little study analyse the role of emotions, and lack of the laws of emotion converted to group behavior in mass emergencies.

Biao Song etal (2014) establish a network model based on evolutionary game theory to study the participants emotional oriented and behavioral evolution direction[6].Chun Wang (2014), using the GARCH-M model, analyze the investor sentiment and the volatility of stock market returns[7]. Congdong Li etal (2013) applied the system dynamics to divide the into three levels, such as psychological, social communication and the way of control of negative emotions. They analyze and simulate the behavior patterns and key variables[8].

In practice, the emotional impact on mass emergencies principal parts is not a single top-down or bottom-up path.The impact of emotion often combined macro and micro. Emotion has an effect on individuals, organizations-behavior or groups-behavior. In this paper, considering the interaction between macro and micro mood, we discuss emotional probable modes of action. Through empirical analysis the emotional impact of mass emergencies behavior, this study can be used as Mass Emergencies factors found in depth. This dissertation help reveal the evolution of mass emergencies inherent laws, guiding the mass emergency response measures to develop a very important significance 


\section{Mass Emergencies Emotional Conduction Network Model}

As one of the important factors that affect the emotional behavior of Mass Emergencies is important to analyze the conduction of the Government's emergency management. At present, the theory of group behavior are many, such as social theory, convergence theory, emergency gauge theory, theory of infection, characterized by social theory, the four elements and group pressure theory. Above theories acknowledge the role of emotion in the generation process of group behavior, but did not analyze the mechanism of mood, so this paper for mood conduction model building based on the above theory and the explore the mechanism of group behavior.(1) Emotional conduction. Conduction process emotions including emotional accumulation, evoking emotion, emotional contagion, emotional intensification and emotional release, the main groups involved in Emergencies will be based on their own experience to respond to changes in the external environment, "relative deprivation" will lead to an individual by naturalization Depending on their mechanism contrary to its desired portion explained, this is the first emotional accumulation. After the accumulation of public sentiment will take the initiative to break through the critical point and communication channels to find emotional release that emotional social share, emotional micro level in this state have gradually transition to the macro level, but also on the emotional mood group members within the group to produce the corresponding strengthening the role of, encountered fuse will lead to mass incidents that sentiment with modern media tools as a carrier, temporarily formed between the main groups involved in incidents of spontaneous self-an instant mood network, with group members embedded networks spread rapidly diffused. Because society actively share of negative emotions have no significant effect on the elimination of the weakening of, so mass events evoking emotion is the basis for mass emergencies, when the group burst incident will affect the mood of participants around people, so that they have the infection under the same emotions, thus completing the shift from the individual to the group. Sentiment intensified so increasing trend of group behavior, from initial development to final peace petition violence. After experiencing the emotional intensification, participants get emotional release, attribution of conduct peaceful, mass emergencies finally be resolved. (2) The behavioral imitation. Mass emergencies, the main groups in the embedded state is likely to be in anomie, homogeneity among group members is among the members of the group to enhance the emotional connection, consistency groups emotional consistency group behavior is usually caused by the activities. "Emergency gauge theory" that the majority of members of the group should have a common action and thus display a common reaction, then group pressure to produce, "suggesting that effect," "demonstration effect" and the "relative deprivation" and other factors work together to make personal feel the need to comply, must comply with group behavior, and I believe the behavior of groups of legitimacy, which is a model once the violence, violence will guide the whole group, group sex incident will be difficult to control.(3) Deindividuation. Depersonalization refers to individuals in a population to reduce the level of control the phenomenon, in this case, the individual will lose their sense of responsibility that their behavior will not have serious consequences, and therefore do not normally make affect the entire group behavior.

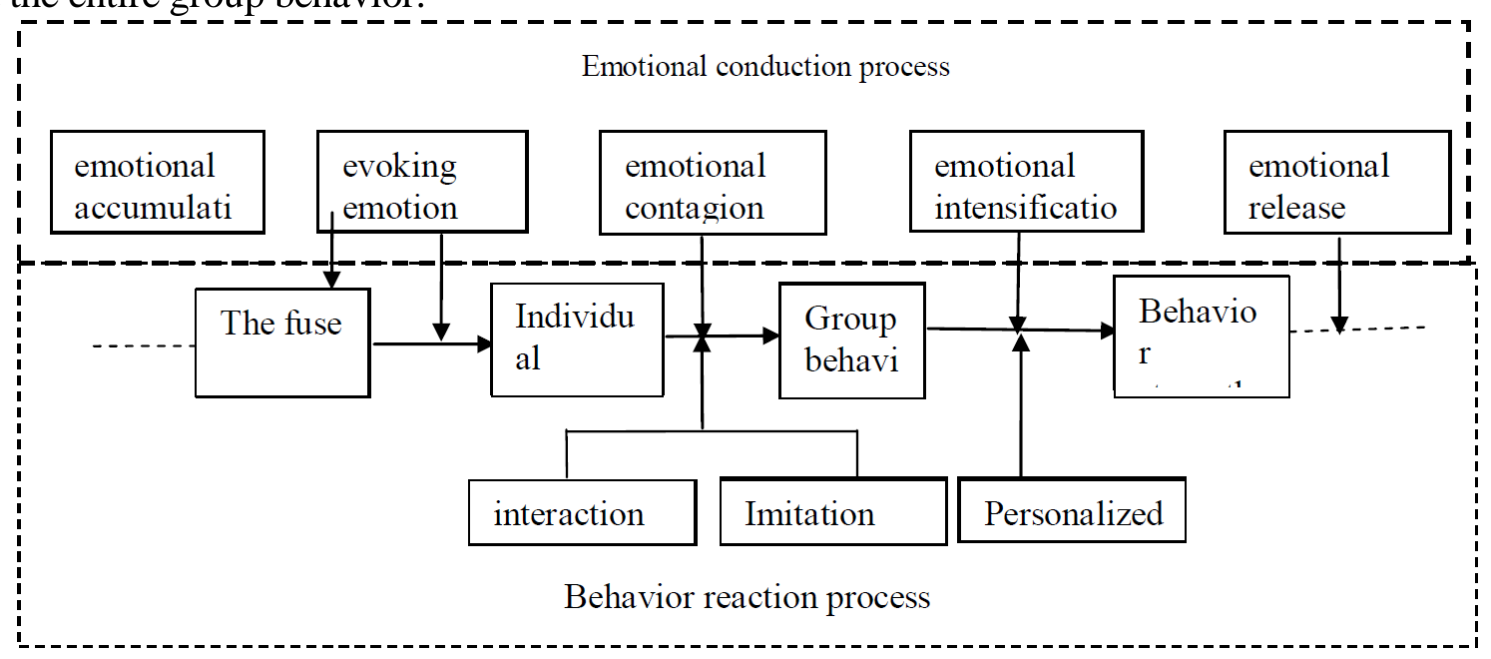

Fig. 1 Emotional transmission network model of Group Emergencies 
The above analysis can be found that the mood of the group members judged by conduction from the external environment. Figure 1 is the Conduction network model, which is comprehensive description of the whole process of emotional conduction.

It can be seen from Figure 1, the produce mechanism based on emotional conduction behavior has undergone a process of emotional accumulation, evoking emotion, emotional contagion, emotional intensification and emotional release.

\section{Empirical Analysis Of The Emotional Impact Of Mass Emergencies Behavior}

To further verify the mood conduction model constructed and their impact on behavior, the paper decided to adopt the way of specific empirical analysis of the impact of mass emergencies emotional behavior. Finucance etal (2000) proposed the emotional heuristics that mood affect risk perception. The study show risk perception depends on the change of mood. Emotion will have a direct impact on the decision-making [9].Rebecca and Chien (2006) study the relationship between emotion and risk perception by experimental. The results indicate that the holder of optimism make conservative decisions, they are averse individual, while holding pessimism those who prefer the relative risk. They further explained, in the face of risk, that people with positive emotions relative to the negative members prefer to take risk-aversion decision [10].

Research Assumption. Assumptions: emotions will affect the behavior of participants in the decision-making choices.

Optimism will reduce the risk perception, the participants showed more conservative, and pessimism will increase participants' risk perception, and their behavior will be more violence. Therefore, the assume will be divided into two sub-hypothesis.

H1: Optimism will participate in the main groups of emergencies tend to be conservative decision-making behavior.

$\mathrm{H} 2$ : Pessimism will participate in the main groups of emergencies tend to be risky decision-making behavior.

Experimental Design and Data Acquisition. By reading the literature, we found that many researchers obtain experimental emotion by induced. Experimentally induced mood has become one of the standard methods study. Evoked emotional material law and Emotional situation evoked France are the two widely used Mood Induction Methods. Materials evoked emotions evoked Law also includes text, images evoked law, music, and odor-induced method. Situations-induced are more complex, including computer games, game play and induced expression / position feedback evoked. In this paper, we choose the text-induced method to complete the experiment. Text-induced method is the way of words to convey the appropriate information to induce subjects to produce the desired experimental mood. In this paper, after analyzing mass incidents occurred in recent years, we develop a questionnaire matched the experiment. In order to ensure the accuracy of the test, we choose emotional self-rating scale proposed by Watson and Tellegen with high consistency and reliability (Consistency coefficient 0.87 ). Subjects will experience the emotions and reflect in the scale, which numbers 1-5 indicate the level of emotions gradually increasing.

Tab. 1 Emotional Self - Rating Scale

\begin{tabular}{cccccc}
\hline & sligh & Alittle & Moderate & Stronge & more Stronger \\
\hline Happy & 1 & 2 & 3 & 4 & 5 \\
Fear & 1 & 2 & 3 & 4 & 5 \\
Afraid & 1 & 2 & 3 & 4 & 5 \\
Sad & 1 & 2 & 3 & 4 & 5 \\
Excited & 1 & 2 & 3 & 4 & 5 \\
Angry & 1 & 2 & 4 & 5 \\
Calm & 1 & 2 & 3 & 4 & 5
\end{tabular}

Happy, excited belong to the category of optimism in the Table 1. Fear, fear, sadness, anger belongs to the category of pessimism. Subjects to rate their extent of pleasure and degree of anger, combined with follow-up questions to determine the relationship between emotion and Mass Emergencies behavior. Under the different conditions of emotions, the score for each option is not the same. Under the conditions of optimism, option A is 5 points, and option $\mathrm{B}$ is 3 points,and option $\mathrm{C}$ is 1 points. In pessimism questionnaire, $\mathrm{A}$ is 5 points, and option $\mathrm{B}$ is 3 points, and option $\mathrm{C}$ is 1 points. In the pessimism of the experiment, if the subject with higher score, it is possible to take a more radical means adventure. 
Experimental Research Methods. For a more realistic reflection of the emotional state of decision-makers, we select society ordinary people to complete the questionnaire. The main object is randomly selected from the society. We grant 80 copies of the optimism questionnaire and 80 copies of the pessimistic questionnaire. After weed out defective questionnaires, there are 103 questionnaires for analysis.

Tab. 2 Statistics Questionnaire

\begin{tabular}{|c|c|c|c|c|c|}
\hline Category & $\begin{array}{c}\text { Questionnaire } \\
\text { issued }\end{array}$ & $\begin{array}{c}\text { Questionnaires } \\
\text { collected }\end{array}$ & $\begin{array}{c}\text { Valid } \\
\text { questionnaires }\end{array}$ & Recovery & Efficiency \\
\hline Quantity (parts) & 160 & 120 & 103 & $75 \%$ & $85.8 \%$ \\
\hline
\end{tabular}

To understand the correlation between emotion and decision, as shown in Table 3,we do the consistency test to analyze the Cronbach coefficient.

Tab. 3 Reliability value of the reference range (Cronbach coefficient)[26]

\begin{tabular}{|c|c|}
\hline Cronbach $\alpha$ coefficient $\leq .300$ & Untrusted \\
\hline $.300<$ Cronbach $\alpha$ coefficient $\leq .400$ & Barely credible \\
\hline $.400<$ Cronbach $\alpha$ coefficient $\leq .500$ & Slightly more credible \\
\hline $.500<$ Cronbach $\alpha$ coefficient $\leq .700$ & Credible \\
\hline $.700<$ Cronbach $\alpha$ coefficient $\leq .900$ & Very credible \\
\hline $.900<$ Cronbach $\alpha$ coefficient & Extremely credible \\
\hline
\end{tabular}

In this paper, the Pearson correlation coefficient was adopted to further measure the correlation between variables. Different Coefficient values represented different relationships. If the coefficient value is greater than zero, then there is a positive correlation between the two variables. If the coefficient value is less than zero, there is a negative correlation between the two variables. As shown in Table 4.

Tab. 4 The reference range of the correlation coefficient [27]

\begin{tabular}{|c|c|}
\hline The correlation coefficient $=1$ & Fully correlated \\
\hline $0.7 \leq$ The correlation coefficient $<1$ & Highly relevant or strong correlation \\
\hline $0.4 \leq$ The correlation coefficient $<0.7$ & Moderate correlation \\
\hline The correlation coefficient $<0.4$ & Low correlation or weak correlation \\
\hline The correlation coefficient $=0$ & Irrelevant \\
\hline
\end{tabular}

This paper uses data SPSS16.0 optimism induced by 50 questionnaire was analyzed. Tables 5 and 6 is pessimistic questionnaire participants under the induced emotion value and the statistics of the decision table.

Tab. 5 optimism induced emotion value statistics

\begin{tabular}{|c|c|c|c|c|}
\hline effective & frequency & percent & valid percent & cumulative percentage \\
\hline \multirow{2}{*}{1} & 12 & 24.0 & 24.0 & 24.0 \\
\cline { 2 - 5 } 3 & 20 & 40.0 & 40.0 & 64.0 \\
\cline { 2 - 5 } 5 & 18 & 36.0 & 36.0 & 100.0 \\
\cline { 2 - 5 } total & 50 & 100.0 & 100.0 & \\
\hline
\end{tabular}

As we can see in table 5, in optimism induction experiments, we get 20 questionnaire score of 3, this number accounts for $40 \%$ of total optimism induced questionnaire, the score of 3 and 5 questionnaires are 44 , accounted for $76 \%$ of the optimism induced by questionnaires, it shows that optimism played a role.

Through spss software to get cronbach 's alpha coefficient is 0.073 , the standardized cronbach's Alpha coefficient is 0.837 , were greater than 0.5 , it explained that between induced emotion value and decision has credible internal consistency. Next, analyze their correlation.

It can seen from table 7, in the trial of optimism induce, decision points and mood scores of Pearson Correlation was 0.728 , it shows that decision points and emotional score has a strong positive correlation. Through the above analysis, we proved the hypothesis of hypothesis 1 . 
Tab. 6 optimism induced emotion value statistics

\begin{tabular}{|c|c|c|c|c|}
\hline effective & frequency & percent & valid percent & cumulative percentage \\
\hline 6.00 & 4 & 8.0 & 8.0 & 8.0 \\
\hline 8.00 & 4 & 8.0 & 8.0 & 16.0 \\
\hline 10.00 & 3 & 6.0 & 6.0 & 22.0 \\
\hline 12.00 & 2 & 4.0 & 4.0 & 26.0 \\
\hline 14.00 & 2 & 4.0 & 4.0 & 30.0 \\
\hline 16.00 & 4 & 8.0 & 8.0 & 38.0 \\
\hline 18.00 & 4 & 8.0 & 8.0 & 46.0 \\
\hline 20.00 & 5 & 10.0 & 10.0 & 56.0 \\
\hline 22.00 & 6 & 12.0 & 12.0 & 68.0 \\
\hline 24.00 & 6 & 12.0 & 12.0 & 80.0 \\
\hline 26.00 & 3 & 6.0 & 6.0 & 86.0 \\
\hline 28.00 & 4 & 8.0 & 8.0 & 94.0 \\
\hline 30.00 & 3 & 6.0 & 6.0 & 100.0 \\
\hline total & 50 & 100.0 & 100.0 & \\
\hline
\end{tabular}

Tab. 7 the correlation analysis of optmistic induced emotion and decision making

\begin{tabular}{|c|c|c|c|}
\hline & & emotion & decision \\
\hline \multirow{3}{*}{ emotion } & Pearson correlation & 5 & $.728^{* *}$ \\
\hline & Significanc(on both side) & & .000 \\
\hline & $\mathrm{N}$ & 50 & 50 \\
\hline \multirow{3}{*}{ decision } & Pearson correlation & $.728^{* *}$ & 5 \\
\hline & Significanc(on both side) & .000 & \\
\hline & $\mathrm{N}$ & 50 & 50 \\
\hline
\end{tabular}

The Analysis of Experimental Results When Public Is Pessimism. In this paper, using SPSS16.0 to analysis through 50 pessimism induced data obtained by questionnaire, tables 8 and 9 is pessimistic questionnaire participants under the induced emotion value and the statistics of the decision table.

Tab. 8 pessimistic induced emotion value statistics

\begin{tabular}{|c|c|c|c|c|c|}
\hline \multicolumn{2}{|c|}{ effective } & frequency & percent & valid percent & Cumulative percentage \\
\hline \multirow{10}{*}{ Data } & 10 & 4 & 8 & 8 & 8 \\
\hline & 12 & 3 & 6 & 6 & 14 \\
\hline & 14 & 5 & 10 & 10 & 24 \\
\hline & 16 & 4 & 8 & 8 & 32 \\
\hline & 18 & 4 & 8 & 8 & 40 \\
\hline & 20 & 6 & 12 & 12 & 52 \\
\hline & 24 & 8 & 16 & 16 & 68 \\
\hline & 28 & 10 & 20 & 20 & 88 \\
\hline & 30 & 6 & 12 & 12 & 100 \\
\hline & total & 50 & 100 & 100 & \\
\hline
\end{tabular}

As we can see in table 9, in pessimism induction experiments, we get 22 questionnaire score of 5, this number accounts for $44 \%$ of total pessimism induced questionnaire, the score of 5 and 3 questionnaires are 44 , accounted for $80 \%$ of the pessimism induced by questionnaires, it shows that pessimism played a role.

Tab. 9 Pessimistic induced mood decision value statistics

\begin{tabular}{|c|c|c|c|c|}
\hline effective & frequency & percent & valid percent & cumulative percentage \\
\hline 1 & 10 & 20 & 20 & 20 \\
\hline 3 & 18 & 36 & 36 & 56 \\
\hline 5 & 22 & 44 & 44 & 100 \\
\hline total & 50 & 100 & 100 & \\
\hline
\end{tabular}

In this paper, the experimental results fully proves that the questionnaire has good reliability and validity, optimism and pessimistic questionnaire reliability analysis Cronbach value is greater than 0.5 , has reached the requirements of reliable. The Pearson correlations of emotion and decision-making are 
above 0.7 . On the basis of exploratory experiments verify the hypothesis of the experiment and its assumptions.

\section{Conclusion and Prospect}

First of all, in this paper, through review the related domestic and foreign literature, constructed the emotion conduction model combined with the actual situation of mass emergencies, proposed generation mechanism based on such an event. Secondly, according to this model to put forward the corresponding experiments hypothesis. At last, we analyze the influence of emotion on the choice of participants in the mass emergencies through the questionnaire. Verify the correlation between emotion and cognitive risk decision-making in the mass emergencies.

The behavior choice in the mass emergencies is a complex dynamic system, and affected by many factors, such as emotion, cognition, risk is interacting, and emotional quantitative and measurement is still a complex problem. The research methods and conclusions in this paper can provide a mirror for summarized the behavior trend analysis in mass emergencies, provide a basis for the government to build the mass emergencies prevention and control system, study interaction between emotional impact factors, it need to study deeply to predict emotional trend.

\section{Acknowledgement}

This research is supported by the National Science Foundation (No. 71173171) and Special Research of Shaanxi Provincial Department of Education (14JZ030).

\section{References}

[1]Liu ShangLiang, Shen Huizhang, Li Feng, Zhang Cong.The Dynamic Game Analysis on Cause of Unexpected Mass Incident in Emergency[J]Journal of Systems \& Management, 2012, (02): 201-205.

[2]Liu Dehai, Wang WeiGuo, Xu Weijun, Zhang WeiGuo. Evolutionary Game Anal ysis of Engendering Mechanism for Mass Emergency under Social Structure [J]. Systems Engineering, 2010, 28(6):88-93.

[3]Xiong Guoqiang, Yu Hongmei, Shi A-pin, Liu Xi. Study of Tripartite Game Model on Interest Adjustment of Group Conflict Management Engineering [J]. Journal of UESTC (Social Sciences Edition),2013,(2):13-16.

[4]WeiHaiyan, ZhangGang.Study on The Influence of Emergency Reporting Frame on Audience Cognition-Taking 2013 H7N9 Avian Influenza as An Example[J]. JOURNAL OF BEIJING INSTITUTE OF TECHNOLOGY (SOCIAL SCIENCES EDITION), $2015,(1): 147-150$.

[5]Xu Yinfeng, Liu Dehai. The Subjective Game Analysis for the Root of Mass Unexpected Incident [J]. FORECASTING, 2004 , 23(6): 43-45+9.

[6]Song Biao, Zhu jian-ming, Huang Qi-fa. The internet public opinion grooming model based on cluster dynamics and evolutionary game theory[J].Systems Engineering-Theory \& Practice, 2014, (11): 2984-2994.

[7]Wang chun. The Effect of Investor Sentiment on Return and Volatility of Stock Market-Based on Empirical Study of Net Flow of Open-end Equity Funds [J].Chinese Journal of Management Science, 2014, (9):49-56.

[8]Li Congdong, Hong Yuxian, Xie Tian. Research on the Dynamic Mechanism of Group Negative Emotion Based on System Dynamics [J]. JOURNAL OF INTELLIGENCE, 2013,(5):38-42.

[9]M. Finucane, A. Alhakami, P.Slovic, S.M.Johnson.The Affect Heuristic in Judgments of Risks and Benefits [J].Journal of Behavioral Decision Making. 2000, 13:1-17.

[10]Chien,Rebecca. Emotion's Effects on People's Decision Making[J]. Journal of Behavioral Decision Making.2006,(3):125-136. 